\title{
Recognition Accuracy and Robustness of Fingerprint Templates Generated by Discrete Fractional Sine Transform
}

\author{
Hiroyuki Yoshimura \\ Graduate School of Engineering, Chiba University, Japan
}

\begin{abstract}
The fingerprint template which corresponds to the phase distributions (PDs) of the 1D discrete fractional sine transforms (DFSTs) with various transforms' orders for the grayscale distributions in the transverse lines of the original fingerprint image is proposed. In addition the recognition accuracy and the robustness of the proposed fingerprint templates are evaluated statistically. The condition to realize the high accuracy of fingerprint recognition using the proposed templates has been made clear on the basis of the Receiver Operating Characteristic (ROC) curve and the minimum error rate (MER). The smallest value of the MER became $1.60 \times 10^{-19} \%$. As for the robustness of the proposed templates, it has been evaluated statistically by use of the normalized cross-correlation function (NCF) between the original data and the inverse transformed data of the proposed template under the condition that the transforms' orders are known by an adversary. As a result, the high security of the proposed templates could be indicated.
\end{abstract}

\section{Introduction}

Individual recognition using the biological information has been recently increasing everywhere, for example, in the automatic logging into a PC, the immigration at the airport, and so on. In particular, the fingerprint recognition system has been widely used because of its high reliability and reasonable price [1].

In general, the enrolled fingerprint images are stored as templates in the database referred in the fingerprint recognition process. The information of the templates should be hidden to keep individual biological information secret. Many methods have been recently proposed, for example, the biometric cryptosystems [2][5], cancellable fingerprint templates [6]-[10], and so on. However, in these methods, since the minutia-based templates are basically used, the recognition accuracy tends to become low in comparison with that of the image-based templates.
In order to realize the fingerprint templates with high recognition accuracy and high robustness against attacks, we have proposed a new method for generating templates by use of the intensity distributions of the $1 \mathrm{D}$ fractional Fourier transforms (FRTs) [11] of original fingerprint images $[12,13]$. In our method, the FRTs with different transforms' orders are applied to the 1D data extracted from the 2D original fingerprint image in a specific direction. However, there might be a possibility that the FRT's order in each line of the fingerprint image is revealed, because the extent of the intensity FRT is dependent on the FRT's order.

In this paper, to solve this problem, we propose the fingerprint templates generated by the DFSTs, and indicate the accuracy of the fingerprint recognition by use of the proposed templates. Specifically, first, we compare the amplitude distribution (AD) and PD of the discrete FRTs (DFRTs), the discrete fractional cosine transforms (DFCTs) [14] and the DFSTs [14] of the fingerprint image, and decide the most appropriate distribution and transform used in the generation process of the proposed fingerprint templates. Next, we evaluate the recognition accuracy of the generated fingerprint templates from the viewpoints of the ROC curve, which denotes the relationship between the false acceptance rate (FAR) and the false rejection rate (FRR), and the MER, where the FAR and the FRR take the same value [15]. In the analysis, the effects of the misalignment in the recognition process are not considered. Finally, we quantitatively analyze the robustness of the templates.

\section{DFRT, DFCT and DFST}

The FRT [11], which is regarded as the generalization of a conventional Fourier transform (FT), was introduced by Namias to solve the problem of a harmonic oscillator in quantum mechanics in 1980 [16]. The FRT of 1D input data $u(x)$ is defined as [11]

$$
\begin{aligned}
u_{p}\left(x_{p}\right)= & \int u(x) \exp \left[i \pi\left(x_{p}^{2}+x^{2}\right) / s \tan \phi\right] \\
& \times \exp \left[-2 \pi i x_{p} x / s \sin \phi\right] d x,
\end{aligned}
$$


where $\phi=p \pi / 2, p$ being the FRT's order, and $s$ is a constant. When $p$ takes a value of $4 n+1, n$ being an integer, the FRT data $u_{p}\left(x_{p}\right)$ corresponds to the conventional FT data. Equation (1) can be easily calculated numerically by a computer [17] or realized experimentally by an optical system composed of a lens and a laser [18].

In general, the DFRT can be expressed in terms of a combination of the DFCT and the DFST, and given by [14]

$$
D F R T_{p}=D F C T_{p}+\exp (-i p \pi / 2) D F S T_{p},
$$

where $p$ denotes the FRT's order, the FCT's order and the FST's order simultaneously. We call $p$ the transform's order in the following.

\section{Method for generating fingerprint templates}

Figure 1 shows two examples of the fingerprint images with 256 by 256 pixels and 500 dpi, which are extracted from the center of the ones used in the Finger Verification Contest held in 2002 (FVC2002) [1]. In order to decide the appropriate templates, we performed the DFRTs, the DFCTs and the DFSTs in the transverse lines (blue lines) of the extracted fingerprint images shown in Figure 1. However, the transforms' orders were changed uniformly and randomly in different transverse lines of the image. In Figure 1(a), as an example, the transforms' orders in 10th, 60th and 250th transverse lines are shown by $p_{10}, p_{60}$ and $p_{250}$, respectively, and basically, $p_{10} \neq p_{60} \neq p_{250}$, i.e., $p_{i} \neq p_{j} \quad(1 \leq i, j \leq 256)$ if $i \neq j$. In Figure 1(b), $q$ is used to express the transforms' orders in place of $p$.

As an example, we changed the transforms' orders, $p_{i}$, uniformly and randomly between 0.7 and 1.0, i.e., $0.7 \leq p_{i} \leq 1.0$, for Figure 1(a) and obtained six templates. Figures 2(a), 2(c) and 2(e) are the amplitude distributions (ADs) of the DFRTs, the DFCTs and the DFSTs, respectively. In addition, Figures 2(b), 2(d) and 2(f) are the phase distributions (PDs) of the DFRTs, the DFCTs and the DFSTs, respectively. From these figures, it was found that the volume of data of the DFCTs or the DFSTs is about a half of that of the DFRTs. In addition, the PDs look like random patterns in comparison with the ADs. Therefore, as the fingerprint templates, the PDs of the DFCTs, i.e., Figure 2(d), or the DFSTs, i.e., Figure 2(f), might be appropriate qualitatively.

In the next section, we quantitatively analyze the ADs and PDs in detail to decide the most appropriate template and the generating condition by use of the normalized cross-correlation function (NCF).

(a)

(b)
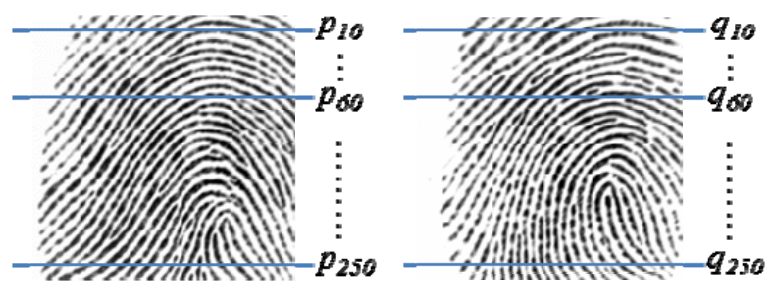

Figure 1. Two examples of the fingerprint images with 256 by 256 pixels and $500 \mathrm{dpi}$, which are extracted from the center of the ones used in FVC2002, and the relationship between the transverse lines in the fingerprint image and the transforms' orders where $p_{10}, p_{60}$ and $p_{250}$ in Figure 1(a) $\left(q_{10}, q_{60}\right.$ and $q_{250}$ in Figure $\left.1(b)\right)$ mean the transforms' orders in 10th, 60th and 250th transverse lines, respectively. (a)

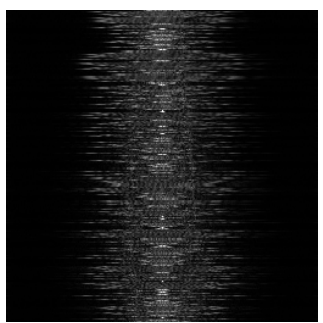

(c)

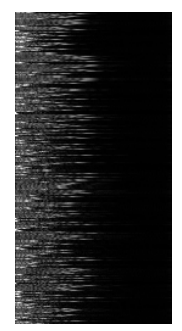

(d)

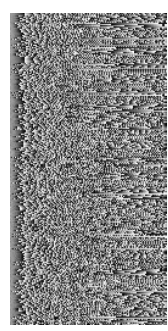

(b)

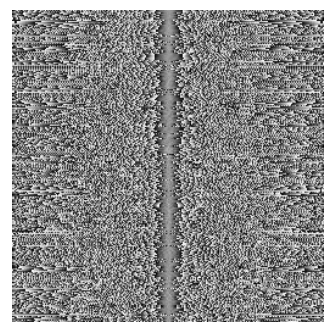

(e)

(f)

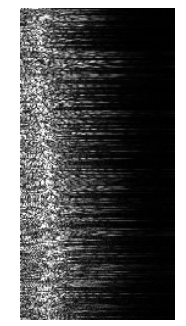

Figure 2. Six examples of the templates obtained from Figure 1(a). The transform' orders, $p_{i}$, were changed uniformly and randomly between 0.7 and 1.0 , i.e., $0.7 \leq p_{i} \leq 1.0$, in different transverse lines of Figure 1(a). (a) ADs of the DFRTs; (b) PDs of the DFRTs; (c) ADs of the DFCTs; (d) PDs of the DFCTs; (e) ADs of the DFSTs; (f) PDs of the DFSTs. 


\section{Comparisons of templates generated by the DFRTs, the DFCTs and the DFSTs}

We generate the ADs and PDs of the DFRTs of two extracted fingerprint images with 256 by 256 pixels, and compare the peak value (PV) of the NCF between the two ADs with that of the NCF between the two PDs. The PV of the NCF is defined as

$$
P V=\frac{\sum_{m=1}^{M} \sum_{n=1}^{N} D_{1}(m, n) D_{2}(m, n)}{\sqrt{\sum_{m=1}^{M} \sum_{n=1}^{N} D_{1}^{2}(m, n)} \sqrt{\sum_{m=1}^{M} \sum_{n=1}^{N} D_{2}^{2}(m, n)}},
$$

where $D_{1}$ and $D_{2}$ denote the generated two ADs or two PDs, and $M$ and $N$ are 256. Similarly, for the DFCTs and the DFSTs, we compare the PVs of the NCFs. In Equation (3), $M$ is 256, but $N$ is 129 for the DFCTs and 127 for the DFSTs. In addition, we analyze the behavior of the PVs by changing the variation ranges of $p_{i}$ and $q_{i}$. Finally, we decide the appropriate distribution (ADs or PDs), the appropriate transform (DFRTs, DFCTs, or DFSTs) and the appropriate variation ranges of $p_{i}$ and $q_{i}$ to generate the most appropriate templates.

4.1 In case of two same fingerprint images, but the transforms' orders are different between the two images $\left(p_{i} \neq q_{i}\right)$

First, we considered the case of two same extracted fingerprint images, that is, Figure 1(b) is the same as Figure 1(a). However, the values of the transforms' orders, $q_{i}$, in the transverse lines were different from those of $p_{i}$, but the variation ranges of $p_{i}$ and $q_{i}$ were the same one.

In the range of $0.1 \leq p_{i} \leq 1.0$ by changing $p_{i}$ uniformly and randomly in different transverse lines of the extracted fingerprint image shown in Figure 1(a), the ADs and PDs of the DFRTs were generated. Moreover, for the same extracted fingerprint image, under the same variation range of $q_{i}$ as $p_{i}$ (i.e., $\left.0.1 \leq q_{i} \leq 1.0\right)$ but the values of $q_{i}$ are different from those of $p_{i}$ (i.e., $p_{i} \neq q_{i}$ ), the $\mathrm{ADs}$ and PDs of the DFRTs were generated. Then, the PV of the NCF between the two ADs or the two PDs could be obtained by use of Equation (3). We repeated the above mentioned process 100 times by changing $p_{i}$ and $q_{i}$ with the same range of $0.1 \leq p_{i}, q_{i} \leq 1.0$, but did not change the extracted fingerprint images, and finally, we obtained the averaged PV (APV) of the NCFs between the ADs of the DFRTs as 0.717. In the case of the PDs, the APV was obtained as $2.24 \times 10^{-2}$.
In a similar fashion, under the same conditions of $p_{i}$ and $q_{i}$ and for the same extracted fingerprint image, we obtained the APV of the NCFs between the ADs or the PDs of the DFCTs or the DFSTs. Moreover, by changing the variation range of $p_{i}$ and $q_{i}$, such as $0.5 \leq p_{i}, q_{i} \leq 1.0$ and $0.9 \leq p_{i}, q_{i} \leq 1.0$, we obtained the APVs of the NCFs between the ADs or the PDs of the DFRTs, the DFCTs or the DFSTs. The results are summarized in Table 1.

As a result, it was found that the APVs of the NCFs of the PDs are extremely low in comparison with those of the ADs. The similar results were obtained for the 109 fingerprint images which were selected one by one from other 109 fingertips in the database used in the FVC 2002. Therefore, as the fingerprint templates, it was found that the PDs of the DFRTs, the DFCTs, or the DFSTs might be appropriate quantitatively in comparison with the ADs.

Table 1. APVs of the NCFs between the two templates, under the condition that $p_{i} \neq q_{i}$, but the variation ranges of $p_{i}$ and $q_{i}$ are the same one between the two same fingerprint images shown in Figure 1(a).

\begin{tabular}{|c|c|c|c|}
\hline $\begin{array}{c}\text { Ranges } \\
\text { of } \boldsymbol{p}_{\boldsymbol{i}}, \boldsymbol{q}_{\boldsymbol{i}}\end{array}$ & $0.1-1.0$ & $0.5-1.0$ & $0.9-1.0$ \\
\hline \hline $\begin{array}{c}\text { ADs of } \\
\text { DFRTs }\end{array}$ & 0.717 & 0.686 & 0.766 \\
\hline $\begin{array}{c}\text { PDs of } \\
\text { DFRTs }\end{array}$ & $2.24 \times 10^{-2}$ & $2.44 \times 10^{-2}$ & $4.77 \times 10^{-2}$ \\
\hline $\begin{array}{c}\text { ADs of } \\
\text { DFCTs }\end{array}$ & 0.721 & 0.721 & 0.752 \\
\hline $\begin{array}{c}\text { PDs of } \\
\text { DFCTs }\end{array}$ & $2.74 \times 10^{-2}$ & $2.97 \times 10^{-2}$ & $5.01 \times 10^{-2}$ \\
\hline $\begin{array}{c}\text { ADs of } \\
\text { DFSTs }\end{array}$ & 0.739 & 0.748 & 0.823 \\
\hline $\begin{array}{c}\text { PDs of } \\
\text { DFSTs }\end{array}$ & $2.26 \times 10^{-2}$ & $2.70 \times 10^{-2}$ & $5.95 \times 10^{-2}$ \\
\hline
\end{tabular}

4.2 In case of two different fingerprint images, but the transforms' orders are same between the two images $\left(p_{i}=q_{i}\right)$

Next, we considered the case of two different extracted fingerprint images, i.e., the case of two different fingertips in the same person or two different persons. However, the transforms' orders, $p_{i}$ and $q_{i}$, in the transverse lines were completely equivalent to each other. This corresponds to the case that, in the fingerprint recognition system, the combination of the values of the transforms' orders in the transverse lines is always fixed for all fingerprint images.

In the range of $0.1 \leq p_{i} \leq 1.0$, by changing $p_{i}$ uniformly and randomly in different transverse lines of 
the extracted fingerprint image shown in Figure 1(a), the PDs of the DFRTs were generated. Moreover, for the different extracted fingerprint image shown in Figure 1(b), under the same condition of $q_{i}$ as $p_{i}$, the PDs of the DFRTs were generated. Then, the PV of the NCF between the two PDs could be obtained. We repeated the above mentioned process 100 times by changing $p_{i}\left(=q_{i}\right)$, but did not change the two extracted fingerprint images, and finally, we obtained the APV of the NCFs between the PDs of the DFRTs as 0.613 .

In a similar fashion, under the same conditions of $p_{i}$ $\left(=q_{i}\right)$ and for the two different extracted fingerprint images shown in Figures 1(a) and 1(b), we obtained the APV of the NCFs between the PDs of the DFCTs or the DFSTs. Moreover, by changing the range of $p_{i}$ and $q_{i}$, such as $0.5 \leq p_{i}\left(=q_{i}\right) \leq 1.0 \quad$ and $0.9 \leq p_{i}\left(=q_{i}\right) \leq 1.0$, we obtained the APVs of the NCFs between the PDs of the DFRTs, the DFCTs or the DFSTs. The results are summarized in Table 2.

As a result, it was found that the APVs of the NCFs between the PDs of the DFRTs or the DFCTs become considerably high. On the contrary, the APVs of the NCFs between the PDs of the DFSTs become extremely low. The similar results were obtained for other 5,994 $\left(={ }_{110} \mathrm{C}_{2}-1\right)$ combinations of the two different extracted fingerprint images, which were obtained from 110 fingerprint images selected one by one from 110 fingertips in the database used in the FVC 2002. Therefore, we can say that the PDs of the DFSTs are the most appropriate template. In the next section, we will investigate the recognition accuracy of our templates which correspond to the PDs of the DFSTs with various transforms' orders in the transverse lines in the extracted fingerprint image.

Table 2. APVs of the NCFs between the two templates under the condition that $p_{i}=q_{i}$ between the two different fingerprint images.

\begin{tabular}{|c|c|c|c|}
\hline $\begin{array}{c}\text { Range of } \\
\boldsymbol{p}_{\boldsymbol{i}}\left(=\boldsymbol{q}_{\boldsymbol{i}}\right)\end{array}$ & $0.1-1.0$ & $0.5-1.0$ & $0.9-1.0$ \\
\hline \hline $\begin{array}{c}\text { PD of } \\
\text { DFRT }\end{array}$ & 0.613 & 0.488 & 0.289 \\
\hline $\begin{array}{c}\text { PD of } \\
\text { DFCT }\end{array}$ & 0.676 & 0.550 & 0.351 \\
\hline $\begin{array}{c}\text { PD of } \\
\text { DFST }\end{array}$ & $6.96 \times 10^{-2}$ & $4.87 \times 10^{-2}$ & $5.50 \times 10^{-2}$ \\
\hline
\end{tabular}

\section{Recognition accuracy of our proposed templates}

In this section, the recognition accuracy of our proposed fingerprint templates is investigated by use of the fingerprint images used in the FVC 2002. First, in subsection 5.1, the behavior of the PV of the NCF between the templates of two different fingerprint images is indicated. Next, the behavior of the PV of the NCF between the templates of the fingerprint images with and without random noise is also indicated. In subsection 5.3, the ROC curves are obtained, and finally, in subsection 5.4, the recognition accuracy of our proposed templates is derived on the basis of the MER [15].

\subsection{Behavior of the PV of the NCF between the templates of two different fingerprint images}

First, in order to obtain the impostor distribution, we analyzed the frequency distribution of the PV of the NCF between templates of two extracted different fingerprint images. We used 110 fingerprint images which were selected one by one from 110 fingertips in the database used in the FVC 2002. Therefore, the total number of frequencies was ${ }_{110} \mathrm{C}_{2}=5,995$.

Figure 3(a) shows the histogram of the PV in case of $0.7 \leq p_{i}\left(=q_{i}\right) \leq 1.0$. The APV is $2.65 \times 10^{-2}$ and the standard deviation of the peak value (SDPV) is $9.92 \times 10^{-3}$. In Table 3 , the APVs and the SDPVs are summarized in cases of $0.5 \leq p_{i}\left(=q_{i}\right) \leq 1.0$, $0.6 \leq p_{i}\left(=q_{i}\right) \leq 1.0 \quad, \quad 0.7 \leq p_{i}\left(=q_{i}\right) \leq 1.0 \quad$, $0.8 \leq p_{i}\left(=q_{i}\right) \leq 1.0$ and $0.9 \leq p_{i}\left(=q_{i}\right) \leq 1.0$. From this table, it was found that the APV takes the smallest value in case of $0.7 \leq p_{i}\left(=q_{i}\right) \leq 1.0$. However, the SDPV takes the smallest value in case of $0.8 \leq p_{i}\left(=q_{i}\right) \leq 1.0$. The condition that both of the APV and the SDPV take the smallest values is desired to achieve the high recognition accuracy. Therefore, we can say that the most suitable condition of $p_{i}\left(=q_{i}\right)$ in our template generating method cannot be decided only from the viewpoint of the impostor distribution.

\subsection{Behavior of the PV of the NCF between the templates of the fingerprint images with and without random noise}

Next, in order to obtain the genuine distribution, we analyzed the frequency distribution of the PV of the NCF between templates of the extracted fingerprint images with and without random noise. Specifically, 110 fingerprint images which had been used in the previous subsection were used. In addition, for each fingerprint image, 50 noise-added fingerprint images were also used. The noise is Gaussian random noise with zero average and the standard deviation of the normalized grayscale of 0.1 . Therefore, the total number of frequencies was 5,500 . 
Figure 3(b) shows the histogram of the PV in case of $0.7 \leq p_{i}\left(=q_{i}\right) \leq 1.0$. The APV is 0.456 and the SDPVs is $3.55 \times 10^{-2}$. In Table 4, the APVs and the SDPVs are summarized in cases of $0.5 \leq p_{i}\left(=q_{i}\right) \leq 1.0$, $0.6 \leq p_{i}\left(=q_{i}\right) \leq 1.0 \quad, \quad 0.7 \leq p_{i}\left(=q_{i}\right) \leq 1.0 \quad$, $0.8 \leq p_{i}\left(=q_{i}\right) \leq 1.0$ and $0.9 \leq p_{i}\left(=q_{i}\right) \leq 1.0$. From this table, it was found that the APV takes the largest value in case of $0.5 \leq p_{i} \leq 1.0$. However, the SDPV takes the smallest value in case of $0.8 \leq p_{i} \leq 1.0$. The condition that the APV takes the largest value and the SDPV takes the smallest value is desired to achieve the high recognition accuracy. Therefore, we can say that the most suitable condition of $p_{i}$ in our template generating

(a)

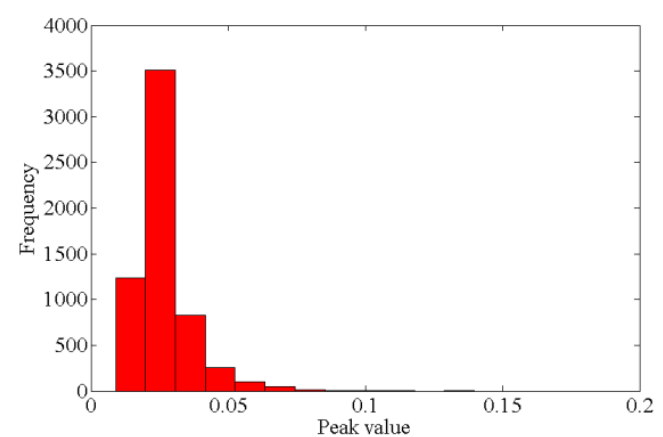

(b)

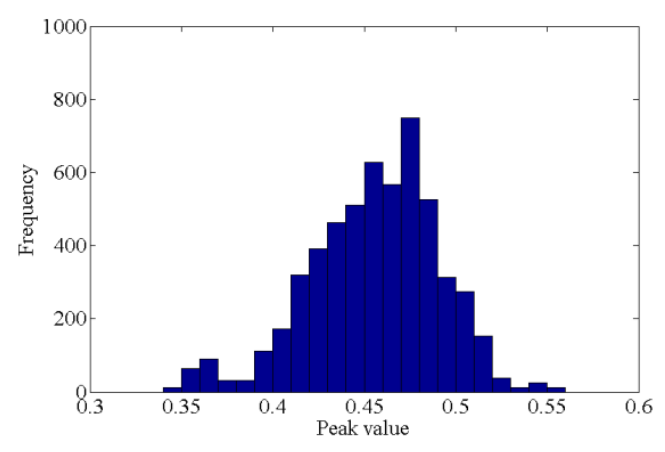

Figure 3. (a) Histogram of the PV of the NCF between the templates of two different fingerprint images in case of $0.7 \leq p_{i}\left(=q_{i}\right) \leq 1.0$. The APV is $2.65 \times 10^{-2}$ and the SDPV is $9.92 \times 10^{-3}$. (b) Histogram of the PV of the NCF between the templates of the fingerprint images with and without random noise, in case of $0.7 \leq p_{i}\left(=q_{i}\right) \leq 1.0$. The APV is 0.456 and the SDPV is $3.55 \times 10^{-2}$.
Table 3. APVs and SDPVs of the NCFs between the templates obtained from two different fingerprint images, in cases of $0.5 \leq p_{i}\left(=q_{i}\right) \leq 1.0 \quad, \quad 0.6 \leq p_{i}\left(=q_{i}\right) \leq 1.0 \quad$, $0.7 \leq p_{i}\left(=q_{i}\right) \leq 1.0 \quad, \quad 0.8 \leq p_{i}\left(=q_{i}\right) \leq 1.0 \quad$ and $0.9 \leq p_{i}\left(=q_{i}\right) \leq 1.0$.

\begin{tabular}{|c|c|c|}
\hline $\begin{array}{c}\text { Range of } \boldsymbol{p}_{\boldsymbol{i}} \\
\left(=\boldsymbol{q}_{\boldsymbol{i}}\right)\end{array}$ & APV & SDPV \\
\hline $0.5-1.0$ & $2.91 \times 10^{-2}$ & $1.41 \times 10^{-2}$ \\
\hline $0.6-1.0$ & $2.73 \times 10^{-2}$ & $1.15 \times 10^{-2}$ \\
\hline $0.7-1.0$ & $2.65 \times 10^{-2}$ & $9.92 \times 10^{-3}$ \\
\hline $0.8-1.0$ & $2.74 \times 10^{-2}$ & $9.37 \times 10^{-3}$ \\
\hline $0.9-1.0$ & $3.51 \times 10^{-2}$ & $1.03 \times 10^{-2}$ \\
\hline
\end{tabular}

Table 4. APVs and SDPVs of the NCFs between the templates obtained from the fingerprint images with and without random noise, in cases of $0.5 \leq p_{i}\left(q_{i}\right) \leq 1.0$, $0.6 \leq p_{i}\left(=q_{i}\right) \leq 1.0 \quad, \quad 0.7 \leq p_{i}\left(=q_{i}\right) \leq 1.0 \quad$, $0.8 \leq p_{i}\left(=q_{i}\right) \leq 1.0$ and $0.9 \leq p_{i}\left(=q_{i}\right) \leq 1.0$.

\begin{tabular}{|c|c|c|}
\hline $\begin{array}{c}\text { Range of } \boldsymbol{p}_{\boldsymbol{i}} \\
\left(=\boldsymbol{q}_{\boldsymbol{i}}\right)\end{array}$ & APV & SDPV \\
\hline \hline $0.5-1.0$ & 0.516 & $4.17 \times 10^{-2}$ \\
\hline $0.6-1.0$ & 0.487 & $3.79 \times 10^{-2}$ \\
\hline $0.7-1.0$ & 0.456 & $3.55 \times 10^{-2}$ \\
\hline $0.8-1.0$ & 0.427 & $3.50 \times 10^{-2}$ \\
\hline $0.9-1.0$ & 0.407 & $3.64 \times 10^{-2}$ \\
\hline
\end{tabular}

method cannot be decided only from the viewpoint of the genuine distribution

\subsection{ROC curve}

As an example, in case of $0.7 \leq p_{i}\left(=q_{i}\right) \leq 1.0$, the impostor and genuine distributions could be obtained from Figures 3(a) and 3(b) by fitting the normalized Gaussian distributions. Figure 4 shows the result. The left-side and right-side curves correspond to the impostor and genuine distributions, respectively. By changing the threshold level corresponding to the PV, the ROC curve could be obtained as shown in Figure 5(a). The results in cases of $0.5 \leq p_{i}\left(=q_{i}\right) \leq 1.0$, $0.6 \leq p_{i}\left(=q_{i}\right) \leq 1.0 \quad, \quad 0.8 \leq p_{i}\left(=q_{i}\right) \leq 1.0 \quad$ and $0.9 \leq p_{i}\left(=q_{i}\right) \leq 1.0$ are also shown in Figures 5(b), 5(c), 5(d) and 5(e), respectively. From these figures, we can understand that the values of the FAR and FRR are the smallest in case of $0.7 \leq p_{i}\left(=q_{i}\right) \leq 1.0$. 


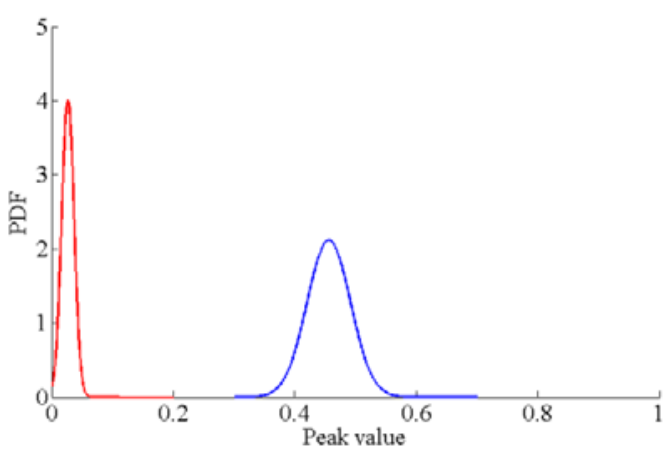

Figure 4. Impostor distribution (left-side curve) and genuine distribution (right-side curve) obtained from Figures 3(a) and 3(b), respectively. PDF means the probability density function.

\subsection{MER}

From the ROC curves shown in Figure 5, we can obtain the MERs. In Table 5, the MERs are summarized in cases of $0.5 \leq p_{i}\left(=q_{i}\right) \leq 1.0$, $0.6 \leq p_{i}\left(=q_{i}\right) \leq 1.0 \quad, \quad 0.7 \leq p_{i}\left(=q_{i}\right) \leq 1.0 \quad$, $0.8 \leq p_{i}\left(=q_{i}\right) \leq 1.0$ and $0.9 \leq p_{i}\left(=q_{i}\right) \leq 1.0$. From this table, it was found that the MER takes the smallest value of $1.60 \times 10^{-19} \%$ in case of $0.7 \leq p_{i}\left(=q_{i}\right) \leq 1.0$. Therefore, we can say that $0.7 \leq p_{i}\left(=q_{i}\right) \leq 1.0$ is the most suitable condition of $p_{i}$ and $q_{i}$ in our template generating method, and our templates can realize the extremely high recognition accuracy, because the smallest values of the FARs and the FRRs of the commercially available fingerprint recognition systems are $0.001 \%$ and $0.1 \%$, respectively, as summarized in Table 2 in [19].

\section{Robustness of our proposed templates}

In this section, the robustness of our proposed fingerprint templates is analyzed by use of the 110 fingerprint images which were used in the previous section. First, for each fingerprint image, 50 templates were generated by changing $p_{i}$ in different transverse lines of the image in $0.7 \leq p_{i} \leq 1.0$. Therefore, we investigated 5,500 cases. Next, the inverse DFSTs (IDFSTs) of the generated templates were performed under the same values of $p_{i}$ which were used in the generating process of the templates. This condition of $p_{i}$ was assumed to be the most serious case that an adversary could get both the template itself and the information about the transforms' orders, $p_{i}$. (a)

(b)
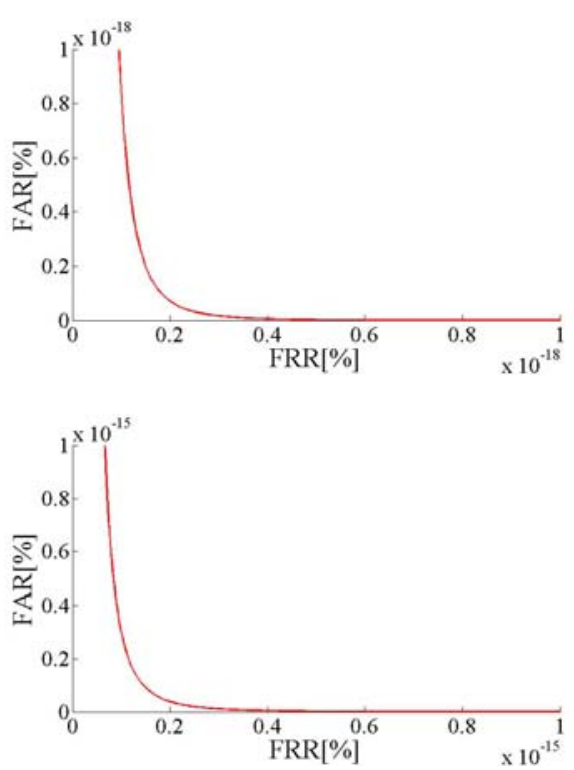

(c)

(d)
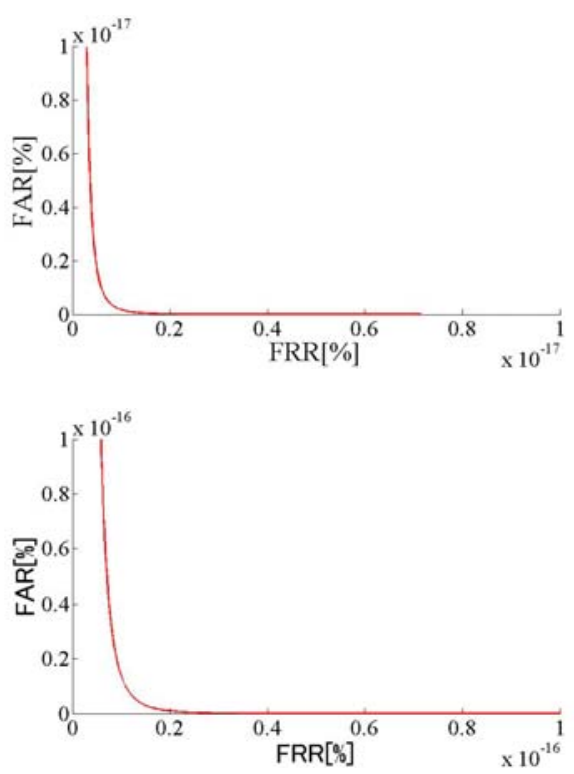

(e)

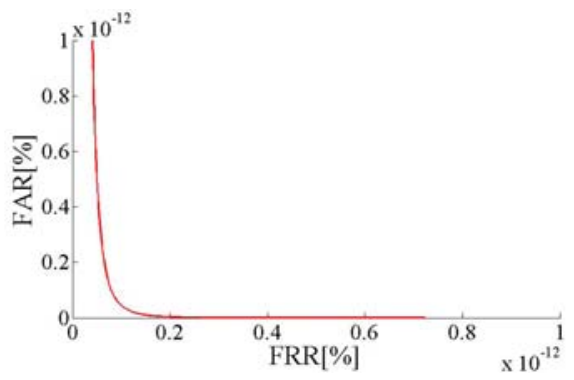

Figure 5. ROC curves in cases of (a) $0.7 \leq p_{i}\left(=q_{i}\right) \leq 1.0 \quad$ (b) $0.5 \leq p_{i}\left(=q_{i}\right) \leq 1.0 \quad$ (c) $0.6 \leq p_{i}\left(=q_{i}\right) \leq 1.0$ (d) $0.8 \leq p_{i}\left(=q_{i}\right) \leq 1.0$ and (e) $0.9 \leq p_{i}\left(=q_{i}\right) \leq 1.0$. 
Finally, we derived the APV and the SDPV of the NCFs between the input data of the DFSTs of the fingerprint images and the obtained IDFST data. As an example, Figure 6(a) shows the input data of the DFST of the fingerprint image shown in Figure 1(a) and Figure 6(b) shows an example of the fingerprint template, i.e., the PDs of the DFSTs. Figure 6(c) shows the obtained IDFST data.

As a result, the APV and the SDPV for 5,500 templates were $4.76 \times 10^{-2}$ and $1.08 \times 10^{-2}$, respectively. For other ranges of $p_{i}$, the APVs and the SDPVs were also obtained and are summarized in Table 6. From this table, it was understood that our templates could not be easily restored to the original input data because the APVs of the NCFs are extremely small. In this way, it was found that our proposed fingerprint templates have fully high robustness.

\section{Conclusions}

In this paper, we have proposed a new method for generating fingerprint templates with high recognition accuracy and high security by use of the DFST. Specifically, the fingerprint template corresponds to the PDs of the DFSTs with different transforms' orders for the grayscale distributions in different transverse

Table 5. MERs obtained from the ROC curves shown in Figure 5.

\begin{tabular}{|c|c|}
\hline Range of $\boldsymbol{p}_{\boldsymbol{i}}$ & MER (\%) \\
\hline $0.5-1.0$ & $1.32 \times 10^{-16}$ \\
\hline $0.6-1.0$ & $6.66 \times 10^{-19}$ \\
\hline $0.7-1.0$ & $1.60 \times 10^{-19}$ \\
\hline $0.8-1.0$ & $1.07 \times 10^{-17}$ \\
\hline $0.9-1.0$ & $8.36 \times 10^{-14}$ \\
\hline
\end{tabular}

(a)

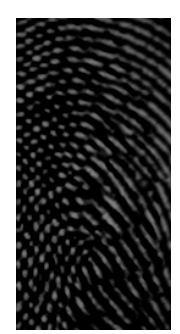

(b)

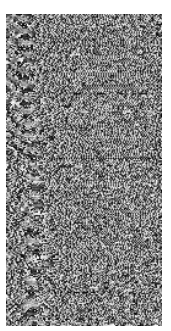

(c)

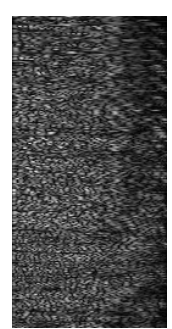

Figure 6. (a) original input data of the DFST of the fingerprint image shown in Figure 1(a), (b) an example of the fingerprint template, i.e., the PDs of the DFSTs and (c) an example of the obtained IDFST data in case of $0.7 \leq p_{i} \leq 1.0$.
Table 6. APVs and SDPVs of the NCFs between the input data of the DFSTs of the fingerprint images and the obtained IDFST data in cases of $0.5 \leq p_{i} \leq 1.0,0.6 \leq p_{i} \leq 1.0$, $0.7 \leq p_{i} \leq 1.0,0.8 \leq p_{i} \leq 1.0$ and $0.9 \leq p_{i} \leq 1.0$.

\begin{tabular}{|c|c|c|}
\hline Range of $\boldsymbol{p}_{\boldsymbol{i}}$ & APV & SDPV \\
\hline \hline $0.5-1.0$ & $5.24 \times 10^{-2}$ & $1.05 \times 10^{-2}$ \\
\hline $0.6-1.0$ & $4.95 \times 10^{-2}$ & $1.06 \times 10^{-2}$ \\
\hline $0.7-1.0$ & $4.76 \times 10^{-2}$ & $1.08 \times 10^{-2}$ \\
\hline $0.8-1.0$ & $4.69 \times 10^{-2}$ & $1.11 \times 10^{-2}$ \\
\hline $0.9-1.0$ & $4.87 \times 10^{-2}$ & $1.19 \times 10^{-2}$ \\
\hline
\end{tabular}

lines of the original fingerprint image. As a result, it was made clear that (i) the recognition accuracy of our fingerprint templates is extremely high, i.e., MER $=1.60 \times 10^{-19} \%$, when $0.7 \leq p_{i} \leq 1.0$; (ii) the robustness, i.e., the security, of our templates is also extremely high.

As a further study, the effects of the misalignment in the recognition process should be analyzed in detail.

\section{References}

[1] D. Maltoni, D. Maio, A. K. Jain, and S. Prabhakar, Handbook of Fingerprint Recognition, Springer, New York, 2003.

[2] U. Uludag and A. Jain, "Securing fingerprint template: Fuzzy vault with helper data," in Proceedings of 2006 Conference on Computer Vision and Pattern Recognition Workshop (CVPRW'06), 2006, pp. 163-170.

[3] A. Nagar, K. Nandakumar, and A. K. Jain, "Securing fingerprint template: Fuzzy vault with minutiae descriptors," in Proceedings of 19th International Conference on Pattern Recognition, 2008 (ICPR2008), 2008, pp.1-4.

[4] K. Xi and J. Hu, "Biometric mobile template protection: A composite feature based fingerprint fuzzy valut," in Proceedings of IEEE International Conference on Communications, 2009 (ICC'09), 2009, pp. 1-5.

[5] R. Wang, X. Yang, X. Liu, S. Zhou, P. Li, K. Cao, and J. Tian, "A novel fingerprint template protection scheme based on distance projection coding," in Proceedings of 2010 20th International Conference on Pattern Recognition (ICPR), 2010, pp. 886-889.

[6] N. K. Ratha, S. Chikkerur, J. H. Connell, and R. M. Bolle, "Generating cancelable fingerprint templates," IEEE Transactions on Pattern Analysis and Machine Intelligence, Vol. 29, No. 4, 2007, pp. 561-572.

[7] C. Lee, J. -Y. Choi, K. -A. Toh, S. Lee, and J. Kim, "Alignment-free cancelable fingerprint templates based on local minutiae information," IEEE Transactions on Systems, Man, and Cybernetics. Part B: Cybernetics, Vol. 37, No. 4, 2007, pp. 980-992. 
[8] H. Yang, X. Jiang, and A. C. Kot, "Generating secure cancelable fingerprint templates using local and global features," in Proceedings of 2nd IEEE International Conference on Computer Science and Information Technology, 2009 (ICCSIT 2009), 2009, pp. 645-649.

[9] B. Yang, C. Busch, P. Bours, and D. Gafurov, "Non invertible geometrical transformation for fingerprint minutiae template protection," in Proceedings of the First International Workshop on Information Forensics and Security, 2009 (WIFS 2009), 2009, pp. 81-85.

[10] Z. Jin, A. B. J. Teoh, T. S. Ong, and C. Tee, "Generating revocable fingerprint template using minutiae pair representation," Proceedings of 2010 2nd International Conference on Education Technology and Computer (ICETC), Vol. 5, 2010, pp. V5-251-V5-255.

[11] H. M. Ozaktas, Z. Zalevsky, and M. A. Kutay, The fractional Fourier transform, John Wiley \& Sons., New York, 2001.

[12] R. Iwai and H. Yoshimura, "Matching accuracy analysis of fingerprint templates generated by data processing method using the fractional Fourier transform," International Journal of Communications, Network and System Sciences, Vol. 4, No. 1, 2011, pp. 24-32. Available: http://www.scirp.org/journal/ PaperInformation.aspx?paperID=3692

[13] R. Iwai and H. Yoshimura, "High-accuracy and highsecurity individual authentication by the fingerprint template generated using the fractional Fourier transform," in Fourier Transforms - Approach to Scientific Principles, (Goran Nikolic, Ed.). InTech, Croatia, 2011, Chapter 15, pp. 281-294. Available: http://www.intechweb.org/books/show/title/fouriertransforms-approach-to-scientific-principles

[14] S. -C. Pei and M. -H. Yeh, "The discrete fractional cosine and sine transform," IEEE Transactions on Signal Processing, Vol. 49, No.6, 2001, pp. 1198-1207.

[15] T. Mansfield, G. Kelly, D. Chandler, and J. Kane, "Biometric Product Testing Final Report, Issue 1.0," in CESG/BWG Biometric Test Programme, Centre for Mathematics and Scientific Computing, National Physical Laboratory, 2001. Available: http://www.lgiris. com/download/brochure/ uk_report.pdf

[16] V. Namias, "The fractional Fourier transform and its application in quantum mechanics," IMA Journal of Applied Mathematics, Vol. 25, No.3, 1980, pp. 241-265.

[17] F. J. Marinho and L. M. Bernardo, "Numerical calculation of fractional Fourier transforms with a single fast-Fourier-transform algorithm," Journal of the Optical Society of America, Vol. 15, No.8, 1998, pp. 2111-2116.

[18] A. W. Lohmann, "Image rotation, Wigner rotation, and the fractional Fourier transform," Journal of the Optical Society of America A, Vol. 10, No.10, 1993, pp. 21812186.

[19] H. Yoshimura, "Optical spatial-frequency correlation system for fingerprint recognition," in State of the Art in Biometrics, (Jucheng Yang and Loris Nanni, Eds.). InTech, Croatia, 2011, Chapter 4, pp. 85-104. Available: http://www.intechweb.org/books/show/title/state-of-theart-in-biometrics 